\title{
Communications
}

2020; 8(1): 1-8

http://www.sciencepublishinggroup.com/j/com

doi: 10.11648/j.com.20200801.11

ISSN: 2328-5966 (Print); ISSN: 2328-5923 (Online)

\section{Exploratory Data Analysis Based on Remote Health Care Monitoring System by Using loT}

\author{
Su Myat Thaung, Hla Myo Tun, Khin Kyu Kyu Win, Myint Myint Than
}

Department of Electronic Engineering, Yangon Technological University, Gyogone, Insein, Yangon Region, Republic of the Union of Myanmar

\section{Email address:}

sumyatthaung.ytu@gmail.com (S. M. Thaung)

\section{To cite this article:}

Su Myat Thaung, Hla Myo Tun, Khin Kyu Kyu Win, Myint Myint Than. Exploratory Data Analysis Based on Remote Health Care Monitoring System by Using IoT. Communications. Vol. 8, No. 1, 2020, pp. 1-8. doi: 10.11648/j.com.20200801.11

Received: November 11, 2019; Accepted: December 25, 2019; Published: January 7, 2020

\begin{abstract}
The system proposed in this article is to make Exploratory Data Analysis (EDA) of people's health condition based on remote health care monitoring system in their different activities. Because of several critical physiological parameters of human body, multi body sensor health monitoring approach is vital. A health care monitoring system is displayed for detecting human physiological information. The system implementation consists of heart beat sensor, temperature sensor and pulse oxygen saturation level (SPO2) sensor. In this framework, gathered health information can be observed by utilizing both PC and cell phones. The real time result of the temperature sensor, heart beat sensor, SPO2 sensor to show on the ThingSpeak is tested. EDA analysis is done on measured data statistic to know the human activity for effective health care monitoring using IoT. The analyses are based on both theoretical approaches and experimental approaches according to the confirmation of recent research outcomes and real data from the hospital. The experimental studies have been carried out based on the idea of medical doctors in the hospital. The technology also confirms that the current research trends would be suitable for real world applications. The confirmation of medical doctors and suggestions on this research works were completed according to the standard of commercial products in the market.
\end{abstract}

Keywords: Internet of Things (IoT), EDA Analysis, Thing Speak, Body Sensor, Remote Health Care System

\section{Introduction}

Things, sensors and gadgets interface and transmit information legitimately and remotely to the Internet by utilizing Internet of Things has getting to be famous with several applications, for example, Health Care System, Smart Home, Smart Farming, Smart Grid, Industrial Internet and so forth. Numerous sensors, microcontrollers, wireless networking, cloud based administrations, versatile applications, website pages and other usage of an IoT applications are associated with this Technologies. The health information of the patient can be observed and constrained by remote health monitoring system.

Present advancements in innovation mainly centre around controlling and observing of various activities. These are progressively rising to arrive at the human needs. The greater part of this innovation is centredaround productive checking and controlling various activities [1].

Thing Speak is an IoT cloud specialist provider. Inserted
IoT gadgets like Arduino, Raspberry Pi can be associated with internet. These sheets at that point can get information or transfer information to Thing Speak storage utilizing APIs. The data stored by a gadget can be gotten to by other customer substances like Mobile, Tablet, PC associated with web utilizing Thing Speak APIs. So in short Thing Speak is an IoT specialist provider that gives APIS to transfer, recover and imagine information from IoT gadgets over cloud [1].

For body sensor node, a great deal of wearable sensors can be utilized. For example, patient position sensor, blood pressure sensor, pulse oximeter sensor, body temperature sensor, airflow sensor, ECG sensor, EMG sensor, EEG sensor, heart beat sensor and SPO2 sensor In this system, heart beat sensor, SPO2 sensor and body temperature sensor are going to use. For each sort of people, the most significant things for a patient are body temperature, pulse and oxygen situation level.

The body should be kept in the ordinary temperature range so that the various procedure of the body can occur 
adequately. Heart rate monitoring is a significant part for a patient and it is a helpful marker to the force of exertion and body's physiological adjustment. If heart failure occurs, it prompts dead. In addition, it is essential to measure the oxygen situation level, as oxygen is indispensable to life and decreased degrees of oxygen coursing in the circulatory system can prompt intense, even lethal difficulties for the heart, lung, brain and different organs. For the above articulations, the entire framework comprises of heart beat sensor, SPO2 sensor and body temperature sensor. In this system all these three sensors will be tested and EDA analysis is done based on the resulting data.

\section{Related Research Works}

In the present remote health care monitoring system are structured by considering diverse human services parameters. Existing framework model is presented in figure 1, Sensors, Zigbee, RFID Tags, Mobile Phone Sensing, WSN Measurement Nodes are utilized for observing of the framework. For information gathering, Microcontroller Unit and WSN Gateway are utilized. Those gathered information will be put away on database and Web Service. There is an Internet interface to remotely screen the gathered information, which is gathered by the sensors, by the Web Browser, Smart Phone and Other Web Based Devices.

A Wireless Sensor Network comprises of numerous modest remote sensors, which are equipped for collecting, storing, processing natural data, and communicating with neighbouring hubs. Before, sensors are associated by wire lines. The access method of WSN gateway hub is helpful on the grounds that information can be received from a WSN by means of the gateway whenever and wherever. A server is an instance of a computer program that acknowledges and reacts to requests made by another program; known as a customer. Less officially, any gadget that runs server programming could be viewed as a server also. Servers are utilized to manage network resources. The services or data in the servers are given through the Internet that are associated through LAN and made accessible for clients by means of smart phones, internet browser or other web browser gadgets to make the system increasingly insightful, versatile and efficient [1].

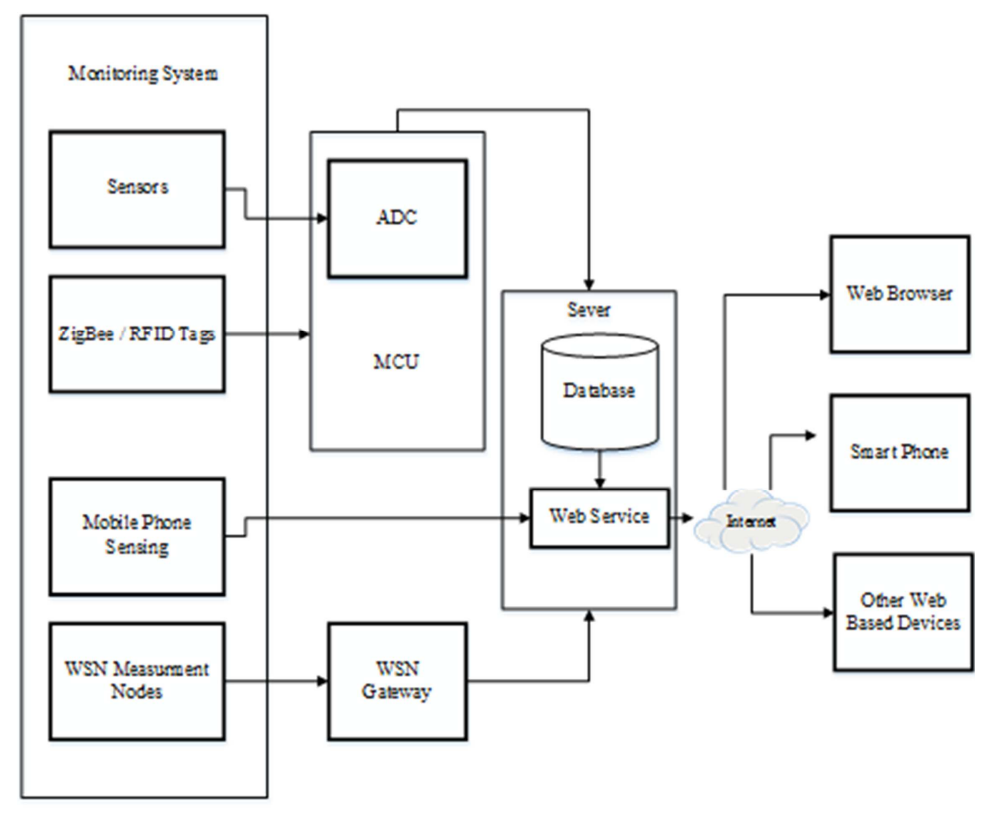

Figure 1. Existing System Model [1].

Remote health-monitoring system contains various sensors which can be used to screen factors and transmit information either to an personal device or to an online storage webpage. These gadgets have the chance to address the patients' issues by overseeing data continuously to the patient's cell phone, $\mathrm{PC}$ or different remote gadgets and can possibly impact their practices. Sensors enable patients to self-screen, track, and survey human physiological information, while additionally giving interfaces and a dashboard for healthcare providers. These sensors are effectively overseen and are winding up progressively exact and solid for patient consideration. The wearable integrated sensor incorporate a wide scope of wearable gadgets and sensors, for example, accelerometers and gyroscopes, smart fabrics and actuators, wireless communication networks and power supplies, and information catch innovation for preparing and choice support [2].

Wearable sensors are used to assemble physiological and development information subsequently empowering patient's status checking. Sensors are conveyed by the clinical use of intrigue. Health monitoring system uses of wearable frameworks frequently utilize numerous sensors that are regularly coordinated into a sensor organize either restricted to bodyworn sensors or integrating body sensors network and encompassing sensors. Uses of wearable sensors and systems that are significant to the field of restoration, for example, 
wellbeing and health observing, security checking, home recovery, appraisal of treatment adequacy, and early recognition of disarranges. Long-term moniroting of physiological information can prompt upgrades in the determination and treatment, for example, of cardiovascular sicknesses. Economically accessible technology provides one with the capacity to accomplish long-term monitoring of pulse, circulatory strain, oxygen saturation, respiratory rate, body temperature and galvanic skin reaction. Several progressing studies are centered around clinically surveying wearable systems created as a feature of significant research projects [3].

A moderately new approach comprises of creating systems dependent on smart sensors. For example, in the Wearable Health Care System project, the system can screen a threelead ECG, electromyogram (EMG) set on the arms, thoracic and abdominal respiration rate, body position and movement, skin temperature, and core temperature. The wearable system incorporates signal-processing module with GPRS or Bluetooth wireless transmission capacities. Another case of utilizing wearable health monitoring system uses to monitor ECG and respiration rate monitoring and a portable electronic board, which assesses the wearer's movement level and is in charge of data transmission from Bluetooth to a nearby PC. Skin temperature sensors are additionally present and the system is intended for the home observing of older individuals or cardiovascular patients. Be that as it may, health monitoring during every day mobile life is likewise possible [4].

This system aims to monitor all kinds of patients. So, body temperature sensor, heart rate sensor and SPO2 sensor are chosen to use and collected data will be store on the cloud by using existing network infrastructure. The body temperature of a normal person is range between $97 \cdot \mathrm{F}$ to $100 \cdot \mathrm{F}$ and the above $100 \cdot \mathrm{F}$ is listed as sick person. Then the heart rate of normal person is $60 \mathrm{bpm}$ to $100 \mathrm{bpm}$ and heart rate of patient is around $120 \mathrm{bpm}$. Moreover, the normal SPO2 level is range between $95 \%$ and $100 \%$ and the value under $90 \%$ is assumed as low SPO2 level. After that EDA analysis is done for human physiological data from resulted data of the remote health care monitoring system.

\section{System Architecture}

In this section, the system architecture of remote health care monitoring system is presented. The system depends on wearable integrated health-monitoring methodology. A wearable gadget is intended to accumulate distinctive biometric information. In the following, the key elements of the system are presented. There are 4 main parts in remote health care monitoring system by using IoT. They are Data Collection, Data Transmission, Data Receiving and Data Monitoring.

EDA is an analysis of understanding about the collected data by utilizing summarizing and visualizing strategies. At a high level state the EDA can be performed in two creases, that is, univariate analysis and multivariate analysis.
Univariate analysis is an examination about individual variables which are analyzed in detachment to have a superior comprehension about those individual variables. Multivariate analysis is attempting to set up a sense of relationship of all factors with each other. In this system, multivariate analysis is used to analysis EAD of human body temperature, heart rate and oxygen saturation level [5].

\subsection{Data Collection}

For data collection, 3 body sensors are used to detect and collect the health data of human. Human Body Temperature Sensor, Heart Rate Sensor and Oxygen Saturation Sensor are used in this system.

Body temperature sensor is attached on the body of human to detect the body temperature of human and it can be vary depens on the hour of day and activities of the person. Various parts of the body have various temperatures. The normal human body temperature is $37.0^{\circ} \mathrm{C}$ which is $98.6^{\circ} \mathrm{F}$. $\mathrm{F}$ Human body temperature can changes only about $0.5^{\circ} \mathrm{C}$, for the duration of the day, with lower temperatures in the first part of the day and higher temperatures in the late evening and night, as the body's needs and due to the activities of human [6].

Oxygen saturation is characterized as the estimation of the measure of oxygen dissolved in blood, in light of the discovery of Hemoglobin and Deoxyhemoglobin. Two diverse light wavelengths are utilized to quantify the real distinction in the assimilation spectra of $\mathrm{HbO} 2$ and $\mathrm{Hb}$. The circulation system is influenced by the centralization of $\mathrm{HbO} 2$ and $\mathrm{Hb}$, and their ingestion coefficients are estimated utilizing two wavelengths $660 \mathrm{~nm}$ (red light spectra) and 940 $\mathrm{nm}$ (infrared light spectra). Deoxygenated and oxygenated hemoglobin retain various wavelengths. Deoxygenated hemoglobin $(\mathrm{Hb})$ has a higher retention at $660 \mathrm{~nm}$ and oxygenated hemoglobin $(\mathrm{HbO} 2)$ has a higher assimilation at $940 \mathrm{~nm}$. At that point a photograph finder sees the nonconsumed light from the LEDs to ascertain the arterial oxygen saturation [6].

The heart rate sensor is a smart wearable device that detects heartbeat from the body to give digital output of heart beat when figure is put on it. This smart instrument utilizes photoplethysmography (PPG) technology and furthermore has two sensors. The main sensor is for recognizing light and another for deciding movement. Its capacity is that the light is illuminated by the skin with a LED and after that the light reflected from the body hits the detector and changes in heartbeat. It takes a shot at the standard of light regulation by blood course through finger at each heart beat [7].

Temperature sensor, heart beat sensor and SPO2 sensor are used to detect patient data. All three sensors are connected to Arduino to collect the detected health data of the patient. In this system, Scheduling Algorithm is used for data collection.

In the Scheduling Algorithm, all the sensors are scheduled to alternate between active and sleep mode. During the idle period sensor node can be switched off. The sensors from the active set are in the active state and all other sensors are in the sleep state. 


\subsection{Data Transmission}

Data transmission refers to the way toward moving information between at least two advanced digital devices.

Data is transmitted starting with one device then onto the next in analog or digital format. Data transmission enables devices or components inside gadgets to address one another. There are two strategies used to transmit information between advanced gadgets: They are Serial Transmission and Parallel Transmission. In this system, serial transmission is proposed to utilize. In parallel transmission the expense just as complexity of the framework increments because of simultaneous transmission of data bits at the same time. Serial transmission mitigates this disadvantage and rises as effective candidate in numerous applications for long distance communication as it diminishes the signal distortion in view of its straightforward structure. Serial transmission is utilized for long distance communication though, the parallel transmission is utilized for shorter separation. Error and noise are least in serial when contrasted with parallel transmission. Serial Transmission is dependable and direct though, Parallel Transmission is questionable and complex. The distortion of a signal is reduced in Serial communication comparing to Parallel communication, so it can transfer data between two separate systems in large distance is possible [8]. Serial transmission is used in this system. Time interval for every sensor is set to be one minute. From the start one minute, heart beat sensor is tested first. After initial one minute, $\mathrm{SPO} 2$ sensor is detected for another one minute. After that temperature sensor is identified for another one minute. Human body temperature may contrast as much as $0.5^{\circ} \mathrm{C}$ $\left(0.9^{\circ} \mathrm{F}\right)$ starting with one day then onto the next. Heart rate will be faster during daily activities or with exercise and other time, it will change slightly. Oxygen saturation level can change for the duration of the day, if a person is transitioning from low-energy to high-energy activities. In this way, taking one minute time interval do not impact on every sensor.

SIM900 GSM-GPRS Shield is an ultra compact and reliable wireless module. SIM900 GSM Module is based on 4 Frequency GPRS module. By using simple AT commands, the GSM-GPRS SIM900 Shield can be configured and controlled via its UART. The GSM/GPRS Modem is having interior TCP/IP stack to empower user to interface with web through GPRS. SIM900 incorporates TCP/IP protocol and expanded TCP/IP AT commands which are helpful for the data transfer applications. It is additionally appropriate for SMS, Voice just as DATA move application in M2M interface [9]. There is super capacitor power supply for the RTC which can work more than 1 day by the power supply of super capacitor. So, when SIM900 module is power off, it can keep the time and day. Features are fully compatible with Arduino UNO and MEGA, Free serial port connecting, Hardware Serial port (D0/D1) or Software Serial port (D2/D3) can be selected to control SIM900 GSM Module. The baud rate is configurable from 9600-115200 through AT command. SIM900 is a quad-band GSM/GPRS module that chips away at frequencies GSM $850 \mathrm{MHz}$, EGSM $900 \mathrm{MHz}$, DCS $1800 \mathrm{MHz}$, and PCS $1900 \mathrm{MHz}$. SIM900 has serial port and debug port which can help client effectively build up client's applications. Audio channel from SIM900 incorporates a microphone and a receiver yield. Is additionally has programmable broadly useful info and yield. The keypad and SPI display interfaces of the SIM900 will give the adaptability to create customized applications [10].

\subsection{Data Receiving}

In efficient receiving algorithm may offer ascent to data loss, processing delay or different issues, and in this way influence the observing of basic parameters. In a Real-Time System (RTS), its capacity must be practiced in the specified time. Polling, Multithreading, timing and different methods can be used for data receiving. Polling Method is the most straightforward algorithm and program structure, which can be accomplished by the loop control structure. Multithreading strategy is multi-stringing can execute various strings simultaneously. In Timing strategy, timer is commonly used data receiving method in real-time monitoring system. In This System, Timing Method is picked to utilize.

\subsection{Data Monitoring}

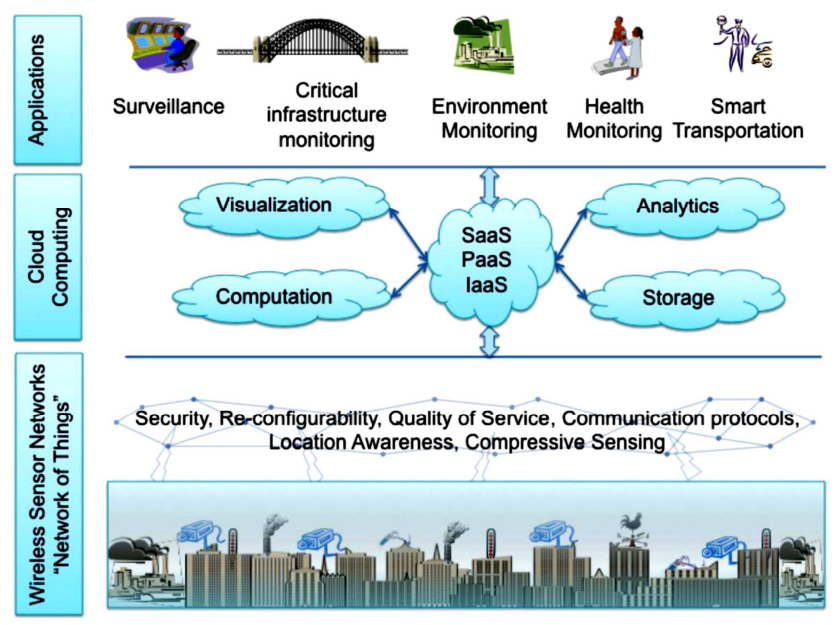

Figure 2. Concept of IoT Frameworkwith Cloud Computing [12].

Receiving data which get from the sensors are display on the ThinSpeak. Collected real time health data can be remotely monitored through ThingSpeak by using both computer and mobile devices.

IoT is a kind of "general worldwide neural network system" in the cloud which associates different things. The IoT is a wisely associated gadgets and frameworks which involved smart machines connecting and communicating with different machines, enviroments, items and infrastructures and the Radio Frequency Identification (RFID) and sensor organize advances will ascend to address this new difficulty. Thus, a tremendous measure of data are being generated, stored and that data is being prepared into valuable activities that can "command and control" the things 
to make lives of people a lot simpler and more secure and to diminish the effect on the earth. Each association, for example, organizations and common establishments needs up-to-date data about people. In such manner, most companyies either use websites, emails or notice sheets. In any case, in the greater part of nations internet access is accessible to individuals on systems and mobile devices of people, with the goal that the moving of the data can be a lot simpler and less expensive through the internet [11].

The concept of IoT framework with cloud computing is demonstrated in figure 2. There are 4 layers. They are Wireless Sensor Layer, Network Layer, Internet Layer and Service Layer. Sensors are associated in system consistently receive and exchange data at Wireless Sensor Layer. Information are gathered from sensors at Wireless Sensor Layer and transfer it to the Network Layer which is known as Cloud Computing Layer. In Network Layer, it incorporates RFID or Bluetooth or Wifi System. There is an Internet Layer to Connect between Network Layer and Service Layer to store data on the cloud. Information originated from Internet Layer is shown in Service Layer which is also known as Application Layer. When the collected data reach to Application Layer, users can easily monitor the resulted data from anywhere at anytime through their computers or mobile devices [12].

\section{Test and Result}

The main focus of this system is testing EDA analysis result of collected health data which appear on the cloud by using Arduino and SIM900. Therefore, hardware experiments are performed with human body temperature sensors, heart rate sensor and oxygen saturation level sensor. To make EDA analysis of different health data, 30 different people of age between 20 to 65 are tested at 5 activities such as sitting, standing, walking, running and climbing in this test and result.

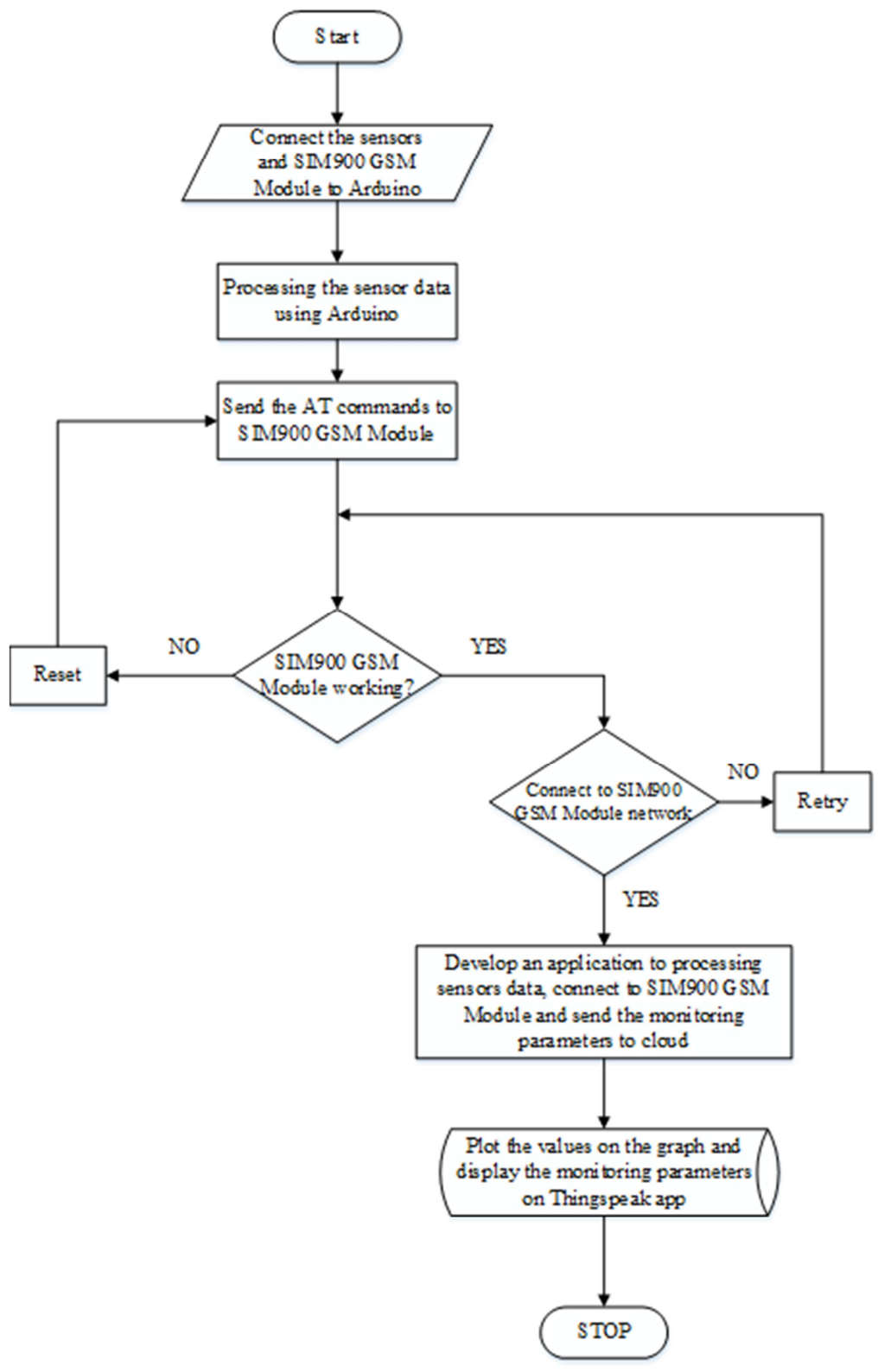

Figure 3. Flowchart of Remote Health Care Monitoring System. 
In this remote healthcare system, Arduino Uno board, human body temperature sensors, heart rate sensor and oxygen saturation level sensor, 4 relay module and SIM900 GSM module are used as an embedded device for sensing and storing the data in to cloud. To collect those patient data, Arduino is used as a microcontroller. Sensors are connected to Arduino Uno board. Its read the sensors and on chip ADC will convert the corresponding sensor reading to its digital value and from that values the realigning heart rate, oxygen saturation level and body temperature of the patient will be assessed. 4 relay module is used to transmit collected health data alternately and simultaneously. SIM900 GSM module interfaces the implanted gadget to web. To show up on the Thing Speak, the gathered health data will be transfer through the internet by using SIM900. To transfer sensors data to end user and furthermore send it to the cloud storage for future utilization, the internet connection has to be established by using SIM900 GSM Module. Flowchart of remote health care monitoring system is shown in figure 3.

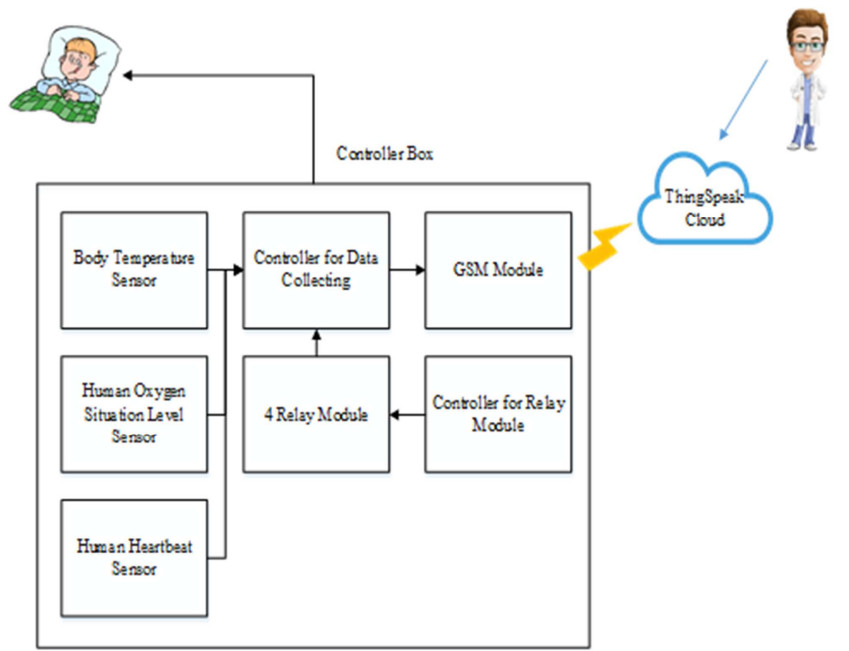

Figure 4. An embedded system designed for health care monitoring system by using 3 body sensors and its components.

An embedded system designed for health care monitoring system by using 3 body sensors and its components are shown in figure 4 . The body sensorsare placed in particular area of a human body for testing purpose. The data result of the collected body sensor is connected to internet through SIM900 GSM module.

Figure 5 shows The hardware testing of healthmonitoring system by using 3 body sensors. The embedded system with its components for reading and to store the result of health data to Thing speak platform (cloud) can be seen in figure 5 . The sensed data will be automatically sent to the Thing speak platform (Cloud), when a proper connection is established with cloud and sensing is successful completed. Then Thing Speak platform analyse and visualize uploaded data. The main purpose of this system is to implement reliable, efficient and low-cost health monitoring system. This system allows patients to be monitored in the comfort of their own homes and get rid of multiple visits to the hospitals.

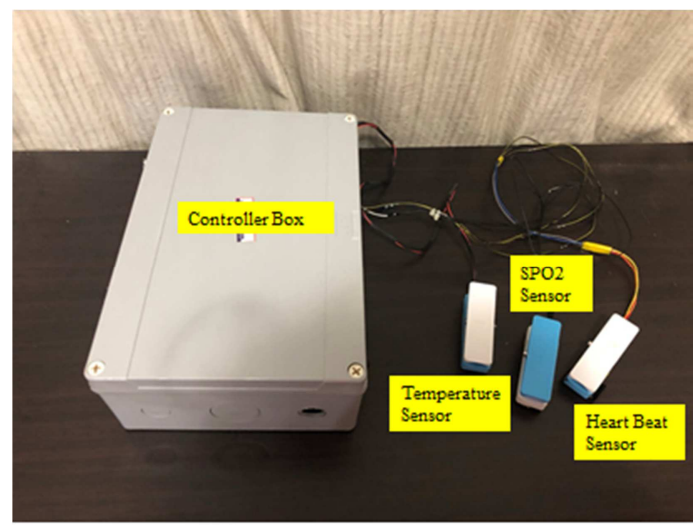

Figure 5. The Hardware Testing of Health-Monitoring System by Using 3 Body Sensors.

After setting up the wearable integrated health-monitoring system by using 3 body sensors, all of 3 body sensors are going to apply on the patient body. As patient data is collected and detected by using body sensor network, doctor can monitor the patient data by using both laptop and mobile applications. An example of the result of the measured health data of a person is described in following figures such as figure 6 , figure 7 and figure 8 .

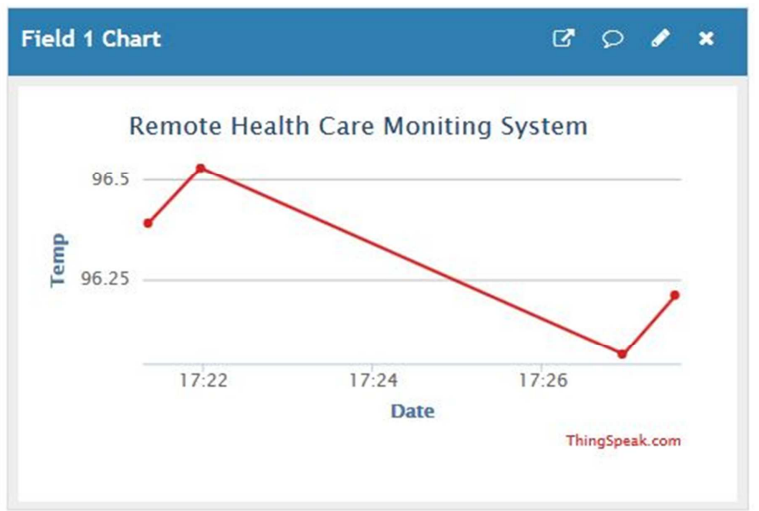

Figure 6. Measured Human Body Temperature Sensor Data Result of a Person.

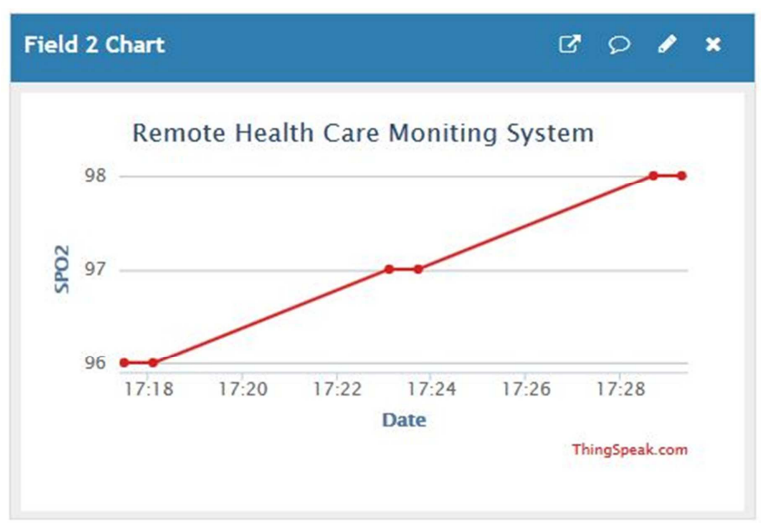

Figure 7. Measured Oxygen Saturation Level Sensor Data Result of a Person. 


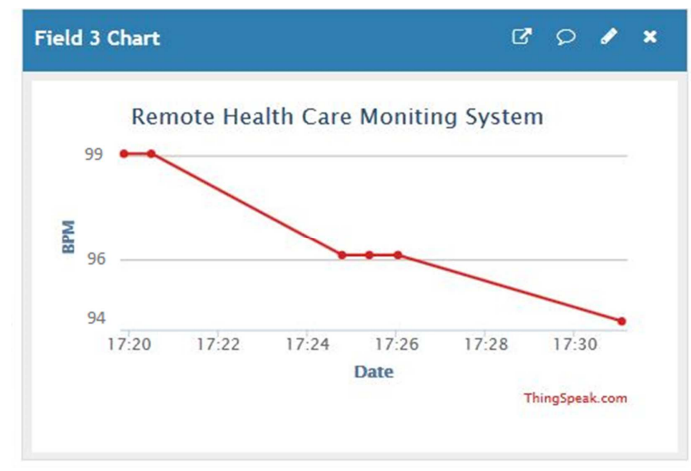

Figure 8. Measured Heart Rate Sensor Data Result of a Person.

After collecting health data result of 30 people at their 5 different activities, EDA analysis is tested and demonstrated in figure (9). Normal healthy oxygen situation level is range between $95 \%$ to $100 \%$. Normal healthy heart rate is range between 60 to $100 \mathrm{bpm}$. Normal healthy human body temperature is $96^{\circ} \mathrm{F}$ to $99^{\circ} \mathrm{F}$. There are different $\mathrm{BPM}, \mathrm{SPO} 2$ and Temperature value for 5 different activities of human as the result shown in figure (10) of Multivariate Analysis.

Correlation Matrix of human heart data at 5 different activities finding from EDA are demonstrated as follow in figure (11), (12), (13, (14) and (15). In Running and Climbing position, there are more heart rate value than other positions. $\mathrm{BPM}$ range is above $125 \mathrm{bpm}$ in those positions, whereas BPM range is between $95 \mathrm{bpm}$ to $88 \mathrm{bpm}$ in other conditions. Body temperature value is between $95^{\circ} \mathrm{F}$ to $98^{\circ} \mathrm{F}$ in all five positions.SPO2 value is between $96 \%$ to $99 \%$ in all five positions. BPM and SPO2 are in a positive relationship in sitting position, that is, $28.19 \%$ of the time BPM increases with increase in SPO2 compare to other positions. Temperature and $\mathrm{SPO} 2$ are in a positive relationship in climbing position, that is, $19.41 \%$ of the time temperature increases with increase in SPO2 compare to other positions. BPM and Temperature are in a negative relationship in running position, that is, $18.42 \%$ of the time increases in BPM will decrease in SPO2 compare to other positions.

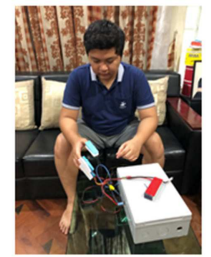

1. Sitting

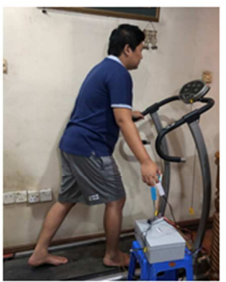

4. Running

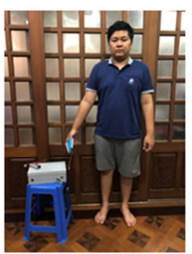

2. Standing

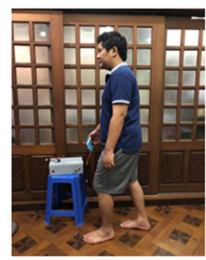

3. Walking

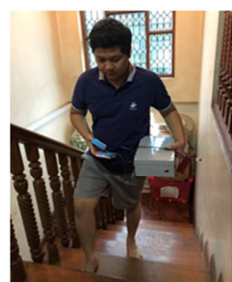

5. Climbing
Figure 9. EDA Analysis in 5 Human Activities.

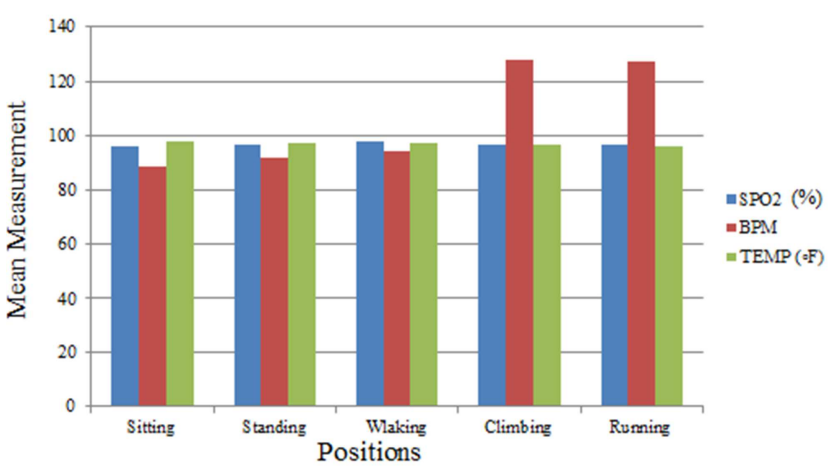

Figure 10. Multivariate Analysis.

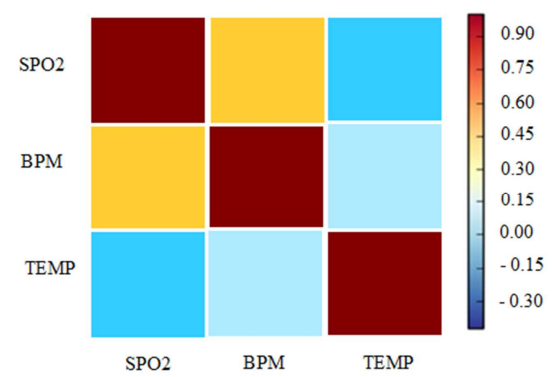

Figure 11. Correlation Matrix of Human at Sitting Position.

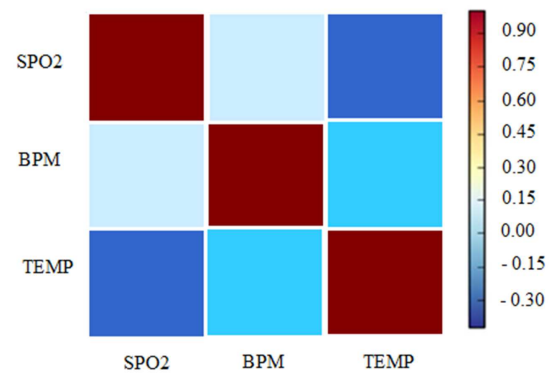

Figure 12. Correlation Matrix of Human atStanding Position.

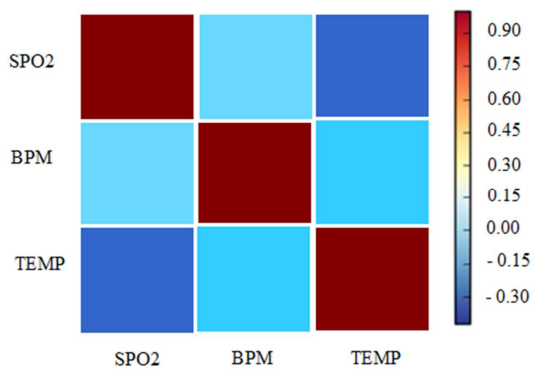

Figure 13. Correlation Matrix ofHuman atWalking Position.

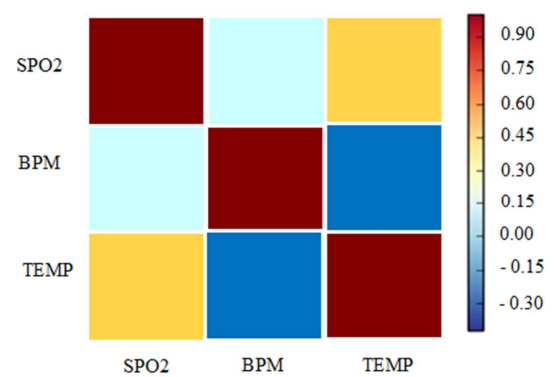

Figure 14. Correlation Matrix of Human atRunning Position. 


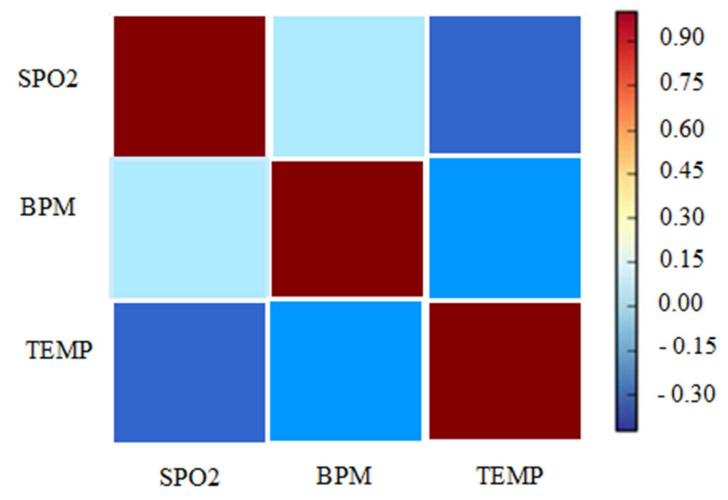

Figure 15. Correlation Matrix of Human atClimbing Position.

\section{Conclusions}

In this paper, wearable integrated health-monitoring system by using 3 body sensors was constructed and EDA analysis is done. The system used Arduino Uno microcontroller, SIM900 GSM Module, human body temperature sensor, heart rate sensor, oxygen saturation level sensor, 4 relays module and Thing Speak. This device makes it possible to perform medical monitoring and possible to use in real- time for monitoring the health state of the human body. In this system, Exploratory Data Analysis is done based on the collected data of 30 people. By seeing the result of sensor data depends on EDA result, we can easily know the activities of that person. From the finding of EDA analysis, the activities of human can be easily differentiated. In future study, the Regression Analysis will do for comparison study of the measured and clinical data can support decision making of the health statistic without assisting the physician. By keeping the embedded devices for the patient to screen their health condition is an incredible system for human. To execute this need to send the body sensor gadgets for gathering the data and analysis. By conveying body sensor devices for human, specialist can realize the healthy state of patient right away. For example specialist and patient can cooperate with other each other through the system. At that point the collected data and analysis results will be accessible to the end client through the internet.

\section{References}

[1] N. Ome and G. S. Rao,"Internet of Things (IoT) based Sensors to Cloud System Using ESP8266 and Arduino Due", International Journal of Advanced Research in Computer and Communication Engineering, vol. 5, issue 10, India, 2016.

[2] G. Appelboom, E. Camacho and M. E. Abraham, "Smart Wearable Body Sensors for Patient Self-Assessment and Monitoring", Arcives of Public Health, 2014.

[3] S. Patel, H. Park and P. Bonato, "A Review of Wearable Sensors and Systems With Application in Rehabilitation", Journal of Neuro Engineering and Rehabilitation, 2012.

[4] F. sanfilippo and K. Y. Pettersen, "A Sensor Fusion Wearable Health-Monitoring System with Haptic Feedback", Aslesund University College, Norway.

[5] M. Swamynathan,"Mastering Machine Learning with Python in Six Steps", Bangalore, Karnataka, India, 2017.

[6] V. Meciak, M. Blaho, L. Mrafko and T. Mudrakova, "SensorBased Platform E-Health connection With Matlab", Slovak University of Technology, Slovak Republic.

[7] H. Shirzadfar, M. S Ghaziasgar, Z. Piri and M. Khanahamadi, "Heart Beat Rate Monitoring UsingOptical Sensors", International Journal of Biosensors and Bioelectronics, vol. 4, issue 2, Iran, 2018.

[8] N. R. Laddha and A. P. Thakare, "A Review on Serial Communication by UART", International Journal of Advanced Research in Computer Science and Software Engineering, vol. 3, issue 1, India, 2013.

[9] M. S. Zaghloul, "Modern Architecutre Tracking System Using Modern GPS Module", International Journal of Scientific and Engineering Research, vol. 5, issue 2, 2014.

[10] M. S. Zaghloul, "GSM-GPRS Arduino Shield (GS-001) with SIM 900 Chip Module in Wireless Data Transmission System for Data Acquisition and Control of Power Induction Furnace", International Journal of Scientific and Engineering Research, vol. 5, issue 4, 2014.

[11] V. Sharma and R. Tiwari, "A Review Paper on "IoT" and It's Smart Applications", International Journal of Scientific and Engineering Research, vol. 5, issue 2, 2016.

[12] J. Gubbi, R. Buyya, S. Marusic and M. Palaniswami, "Internet of Thing (Iot): A Vision, Architectural Elements, and Future Directions", The University of Melbourne, Australia. 